\title{
Logic Operations of Charge Qubit in a Triple Quantum Dot
}

\author{
K. PTASZYŃSKI ${ }^{a, b *}$ AND B.R. BUŁKA ${ }^{a}$ \\ ${ }^{a}$ Institute of Molecular Physics, Polish Academy of Sciences, M. Smoluchowskiego 17, 60-179 Poznań, Poland \\ ${ }^{b}$ Faculty of Technical Physics, Poznań University of Technology, Piotrowo 3, 60-695 Poznań, Poland
}

Studies for a single charge qubit and two capacitively coupled qubits built on triple quantum dots are presented. We show feasibility of implementing two-qubit gate operations, e.g. the CPHASE gate can be implemented with the fidelity higher than $99 \%$ for strong couplings.

DOI: 10.12693/APhysPolA.129.A-30

PACS: 73.21.La, 03.67.Lx

\section{Introduction}

Charge qubits realized in the double quantum dot systems have been a subject of many theoretical and experimental studies due to their simplicity and fast operation speed [1]. However they exhibit short decoherence times caused by charge noise and phonons. Hentschel et al. [2] followed by Vasiliev and Fedichkin [3] showed enhanced robustness to phonon-induced decoherence in the triple quantum dot charge qubit in triangular and linear geometry, respectively.

In this paper we develop the concept of triple quantum dot charge qubit. We show that leakage to a higher energy level, limiting the single qubit gate fidelities, can be eliminated by an appropriate modification of tunneling barriers and dot energies. Next we present studies for two capacitively coupled qubits and show a feasibility of implementing of two-qubit operation gates for two different coupling topologies.

\section{Single qubit}

The charge qubit is defined in an artificial molecule consisting of three coherently coupled quantum dots with a single charge carrier. We assume that the quantum dot energies and the tunnel couplings between them can be changed independently by applying voltages applied to the electrodes. The system is described by the model Hamiltonian

$$
\hat{H}=\sum_{i}^{3} \epsilon_{i} c_{i}^{\dagger} c_{i}+\sum_{i}^{3} \sum_{j>i}^{3} t_{i j}\left(c_{i}^{\dagger} c_{j}+c_{j}^{\dagger} c_{i}\right)
$$

where $\epsilon_{i}$ is an energy level at the $i$-th dot, $t_{i j}$ is a tunnel coupling between the dots $i$ and $j$ and $c_{i}^{\dagger}\left(c_{i}\right)$ is a fermionic creation (annihilation) operator. Because we consider only one charge carrier in the system the spin is neglected. In a fully symmetric case $\left(\epsilon_{i}=\epsilon, t_{i j}=t\right)$ the eigenstates correspond to the states in the Jacobi coordinates [4]: $|0\rangle=(|q 00\rangle-|0 q 0\rangle) / \sqrt{2},|1\rangle=(|q 00\rangle+$ $|0 q 0\rangle-2|00 q\rangle) / \sqrt{6}$ and $|Q\rangle=(|q 00\rangle+|0 q 0\rangle+|00 q\rangle) / \sqrt{3}$,

\footnotetext{
* corresponding author; e-mail:

krzysztof.ptaszynski@ifmpan.poznan.pl
}

where $q$ denotes the position of the charge carrier. We further assume that the charge carrier is a hole (or, equivalently [5], that our system contains five electrons). In this case $t>0$, and the states $|0\rangle$ and $|1\rangle$ form a doubly degenerate ground state. $|Q\rangle$ is an excited state separated by an energy gap $3 t$. We use the states $\{|0\rangle,|1\rangle\}$ to define a computational space of the qubit. To make manipulation of the qubit possible we introduce asymmetry to the system, which lifts the ground state degeneracy. We describe it as an influence of an effective electric field, which modifies the parameters: $\epsilon_{i}=\epsilon+g_{E} \cos (\theta+i 2 \pi / 3)$, $t_{i j}=t+g_{E} \cos (\theta+2(6-i-j) \pi / 3)$. Here, $\theta$ is the angle describing direction of the effective field in respect of the vector starting at $3 \mathrm{rd}$ dot and perpendicular to the opposite base of the triangle and the parameter $g_{E}$ is proportional to the magnitude of the field. Then, the effective Hamiltonian of the qubit subspace can be expressed as

$$
\hat{H}_{q}=C \mathbb{I}_{2}+\frac{\delta}{2} \sigma_{z}+\frac{\gamma}{2} \sigma_{x},
$$

where $C=\epsilon-t, \delta=-3 g_{E} \cos (\theta), \gamma=-3 g_{E} \sin (\theta)$. This is mathematically equivalent to the Hamiltonian of a spin in a magnetic field oriented in the direction $\gamma \hat{x}+\delta \hat{z}$ with a magnitude proportional to $g_{E}$. Tuning the Hamiltonian parameters enables full control of a qubit position on the Bloch sphere.

We modify both quantum dot energies and tunneling couplings in our system, which allows to decouple the computational subspace from the $|Q\rangle$ state: $\langle 0|\hat{H}| Q\rangle=$ $\langle 1|\hat{H}| Q\rangle=0$. Due to this fact, high values of $g_{E}$ can be used, thus increasing operation speed, without any mixing with the $|Q\rangle$ state. This is a serious advantage in comparison with the system considered by Hentschel et al. [2], where only the tunnel couplings were modified and, therefore, mixing terms were present. Let us consider their system with a tunnel coupling detuning by a factor of 10 (which is technically achievable [6]) which corresponds to $g_{E}=0.8$. In this case the Pauli-X gate fidelity (the probability of obtaining a desired final state) is reduced by a leakage to about $96 \%$.

\section{Two qubits}

In this part we consider two triple-quantum dot molecules with capacitative coupling between them. We 
assume that for sufficiently weak couplings the leakage from the computational subspace can be neglected, and therefore the two-qubit system is effectively formed. The coupling between the qubits is described by an interaction Hamiltonian

$$
\hat{H}^{\prime}=\sum_{i=1}^{3} \sum_{j=1}^{3} U_{i j} n_{i}^{1} n_{j}^{2},
$$

where $n_{i}^{1}$ and $n_{j}^{2}$ denote charge number operators at the $i$-th dot of the first and the $j$-th dot of the second qubit, respectively, and $U_{i j}$ is a Coulomb interaction between quantum dots. Our model is qualitatively different from the system studied by Hentschel et al. [2], where a tunnel coupling between the qubits was considered.

(a)

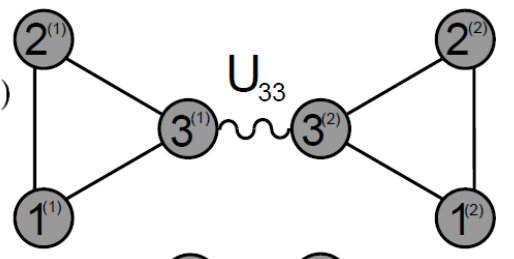

(b)

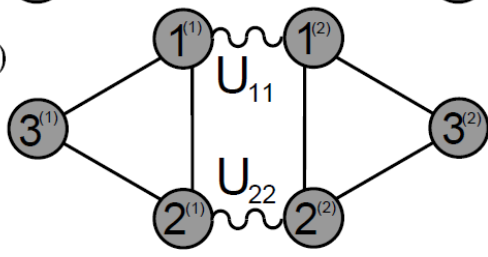

Fig. 1. Tip-to-tip (a) and base-to-base (b) coupling topologies.

We study two coupling topologies: with one coupling (tip-to-tip, Fig. 1a) and two couplings (base-to-base, Fig. 1b). For the tip-to-tip configuration an effective twoqubit Hamiltonian is

$$
\hat{H}_{2 q}=C \mathbb{I}_{4}+\hat{H}_{q}^{1} \otimes \mathbb{I}_{2}+\mathbb{I}_{2} \otimes \hat{H}_{q}^{2}+\hat{H}_{i n t},
$$

where $C=U_{33} / 9, \hat{H}_{q}^{1}$ and $\hat{H}_{q}^{2}$ corresponds to the first and second qubit with modified parameters $\delta^{i}=$ $-3 g_{E}^{i} \cos \left(\theta^{i}\right)-2 U_{33} / 9, \gamma^{i}=-3 g_{E}^{i} \sin \left(\theta^{i}\right) . \quad \hat{H}_{\text {int }}$ describes interactions between the qubits which for this case is of an Ising type

$$
\hat{H}_{i n t}=J_{z z} \sigma_{z}^{1} \otimes \sigma_{z}^{2}
$$

with $J_{z z}=U_{33} / 9$.

For the base-to-base topology and $U_{11}=U_{22}=U$ the parameters are $C=2 U / 9, \delta^{i}=-3 g_{E}^{i} \cos \left(\theta^{i}\right)+2 U / 9$, $\gamma^{i}=-3 g_{E}^{i} \sin \left(\theta^{i}\right)$, and the interaction Hamiltonian

$$
\hat{H}_{\text {int }}=J_{x x} \sigma_{x}^{1} \otimes \sigma_{x}^{2}+J_{z z} \sigma_{z}^{1} \otimes \sigma_{z}^{2}
$$

is of the anisotropic $X Y Z$ Heisenberg type with $J_{x x}=$ $U / 6, J_{z z}=U / 18$. These results are in contrast to the case of two double-dot qubits, where the interaction Hamiltonian is always of the Ising type [1].

Let us now consider how to perform two-qubit operations in these two topologies. We begin with a CPHASE gate, which is a unitary $\operatorname{diag}(1,1,1,-1)$. It can be proved that combination of the CPHASE and a full set of single qubit gates allows us to perform universal quantum computation [7]. For the tip-to-tip topology, realization of this gate is straightforward for $g_{E}^{1}=g_{E}^{2}=0$ due to the absence of non-diagonal elements of interaction Hamiltonian (5). The operation time for this gate is equal to $9 \hbar \pi / J_{z z}$ with $J_{z z}=U / 9$. For the base-tobase topology realization of the CPHASE gate is also possible, however additional single qubit gates are required. Operation time is still $9 \hbar \pi / J_{z z}$, with a smaller value of $J_{z z}=U / 18$, proportional to the diagonal part of the Hamiltonian. However, it can be shortened to $9 \hbar \pi / J_{x x}$, when the Hadamard gates on both qubits are implemented before and after the CPHASE gate implementation.

Although the CPHASE gate is sufficient for universal quantum computation, realization of other specific twoqubit gates in an optimal time and with a minimal operation complexity is worth of considering. One can ask whether the interaction Hamiltonian of the base-to-base topology (6) enables a realization of some specific quantum gates in a shorter time or in a more simple way. It is known that, due to the favorable mathematical form of the Hamiltonian, the symmetric Heisenberg interaction enables straightforward realization of the SWAP gate, while the $X Y$ Heisenberg interaction - of the iSWAP gate [7]. However, in our case, because of the Hamiltonian asymmetry, performing of these gates requires additional single qubit gates (equivalent to a basis rotation). The iSWAP gate, defined as a state vector transformation $(a, b, c, d) \rightarrow(a, \mathrm{i} c, \mathrm{i} b, d)$, can be implemented in a time optimal way using 2 two-qubit operations. During the first step one uses an interaction Hamiltonian in the rotated basis $\hat{H}_{i n t}=U\left(\sigma_{x} \sigma_{x} / 6+\sigma_{y} \sigma_{y} / 18\right)$, in the second one $-\hat{H}_{\text {int }}=U\left(\sigma_{x} \sigma_{x} / 18+\sigma_{y} \sigma_{y} / 6\right)$. Basis rotations require additional 16 single-qubit gates $(8$ for each qubit). A comparable complexity (2 two-qubit and 17 single-qubit gates) is required for realization of a qualitatively similar Bogolyubov gate. Using the Isingtype Hamiltonian, the iSWAP gate or Bogolyubov gate requires twice the time, but with a lower operation complexity - only 1 two-qubit operation and 4 Hadamard gates are required. The Ising-type Hamiltonian allows also to perform the SWAP gate using a single-unitary operation. We conclude that the shorter operation time for the base-to-base topology results from the stronger coupling, which is the consequence of the increase in number of interacting quantum dots. Higher operation complexity results from anisotropy of the interation.

In the case of two qubit operations, leakage from the computational subspace is unavoidable, and it becomes greater as $U$ increases. For the tip-to-tip topology interaction predominantly $|1\rangle|Q\rangle,|Q\rangle|1\rangle$ states are populated due to the leakage, whereas probability of the leakage to $|Q\rangle|Q\rangle$ state is very small and the states $|0\rangle|Q\rangle,|Q\rangle|0\rangle$ are completely decoupled. However, in the particular case of the CPHASE gate, a decrease of the gate fidelity as a function of $U$ is nonmonotonic - local maxima are observed. For example at $U=1.665 t$ the fidelity is $99.44 \%$ (assuming no decoherence), making the gate operation 
feasible even for a non-perturbative coupling between the qubits. This is because the probability of leakage oscillates in time, and it decreases to a small value when the operation is finished. For other types of operation gates one observes a more significant decrease of the fidelity with $U$. A similar reduction of the fidelity occurs also in the case of the base-to-base coupling.

\section{Conclusions}

Summarizing, we have studied the charge qubit built of three coherently coupled quantum dots. An appropriate choice of the parameters allows to avoid leakage beyond the qubit subspace during single qubit gates even for high operation speeds. We also show the feasibility of two qubit gates implementation for two different coupling topologies of capacitative couplings. Different types of gates can be performed by either a single unitary operation or a sequence of operations. In particular, the tipto-tip topology, corresponding to the Ising-type interaction, enables straightforward realization of the CPHASE gate. Moreover, it can be implemented with relatively low leakage (99.44\% fidelity) even for non-perturbative coupling between the qubits. The gate realization using the base-to-base coupling requires a higher number of unitary operations, however may be more time-optimal, if the value of inter-dot Coulombic coupling is limited.

\section{Acknowledgments}

This work has been supported by the National Science Centre under the contract DEC-2012/05/B/ST3/03208.

\section{References}

[1] G. Shinkai, T. Hayashi, T. Ota, T. Fujisawa, Phys. Rev. Lett. 103, 056802 (2009); T. Fujisawa, G. Shinkai, T. Hayashi, T. Ota, Phys. E 43, 730 (2011).

[2] M. Hentschel, D.C.B. Valente, E.R. Mucciolo, H.U. Baranger, Phys. Rev. B 76, 235309 (2007).

[3] A.U. Vasiliev, L. Fedichkin, Quantum Inf. Process. 13, 8 (2014).

[4] C.-Y. Hsieh, Y.-P. Shim, M. Korkusinski, P. Hawrylak, Rep. Prog. Phys. 75, 114501 (2012).

[5] M. Korkusinski, I.P. Gimenez, P. Hawrylak, L. Gaudreau, S.A. Studenikin, A.S. Sachrajda, Phys. Rev. $B$ 75, 115301 (2007).

[6] C.B. Simmons, M. Thalakulam, B.M. Rosemeyer, B.J. Van Bael, E.K. Sackmann, D.E. Savage, M.G Lagally, R. Joynt, M. Friesen, S.N. Coppersmith, M.A. Eriksson, Nano Lett. 9, 3234 (2009).

[7] N. Schuch, J. Siewert, Phys. Rev. A 67, 032301 (2003). 